\title{
Technical possibilities for coronary artery bypass surgery and the outlook for graft patency
}

\author{
M. H. Yacoub \\ F.R.C.S. \\ Harefield Hospital, Middlesex, and National Heart Hospital, London W.1
}

\begin{abstract}
Summary
In an attempt to analyse the results of saphenous bypass grafting, 213 patients were re-investigated by repeat coronary, graft and left ventricular angiography. In this series, the presence of diffuse arterial disease was not considered as a contra-indication to operation. Instead, additional endarterectomy was performed.

The overall patency rate was $88 \%$ and was not significantly altered by the passage of time, although the patency rate during the first 6 months was $94 \%$. The patency rate did not appear to be influenced by the age of the patient, the severity of the lesion pre-operatively, the artery grafted nor additional endarterectomy. The run-off was judged to be good in $82 \%$ of grafts investigated before 6 months, compared to $70 \%$ of those investigated later. Progress of the disease in the native vessels appeared to be slightly accelerated in the segment proximal to the graft.
\end{abstract}

\section{Introduction}

Although myocardial revascularization by saphenous or internal mammary bypass grafting is now established, we believe that at this stage of our knowledge it is essential to continue to evaluate critically the results of these procedures.

The early and long-term results of the operation should depend upon the ability of the inserted grafts to remain patent, and on the changes in the native coronary circulation following grafting. It has been our policy not to regard the presence of diffuse arterial disease nor the angiographic non-visualization of the distal segment beyond the obstruction as contra-indications to operation. In these patients, the vessel is examined at the time of operation and additional endarterectomy is performed if necessary.

The purpose of this paper is to analyse the results of this policy and to attempt to define the early and late behaviour of the grafts and their effect on the native coronary circulation.

\section{Patients and methods}

Two hundred and thirteen patients, who underwent coronary grafts, were re-investigated by repeat coronary, graft and left ventricular angiography. The indication for this was routine re-investigation or recurrence of symptoms. Two hundred and three were male and ten female, with ages varying between 27 and 68 years. The period between operation and investigation varied from 2 weeks to 48 months (mean 20 months). For the sake of analysis, grafts which could not be entered were considered occluded. The run-off was graded into good, moderate or poor by two independent observers. The operative technique, with or without endarterectomy, has been described elsewhere (Yacoub et al., 1974, 1975).

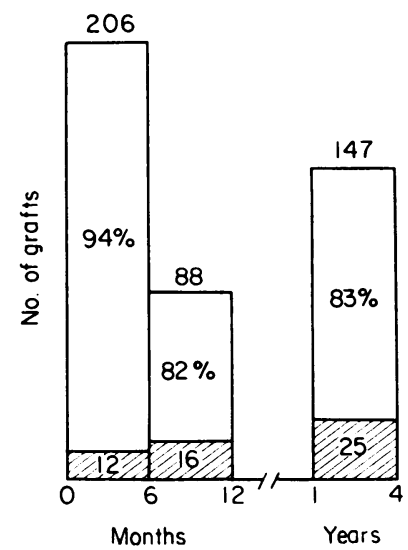

Fig. 1. Patency rate as related to time since operation. Total 441: $\mathbb{Z}$, not entered 5 , occluded $48 ; \square$, patent 388. Patency rate $88 \%$.

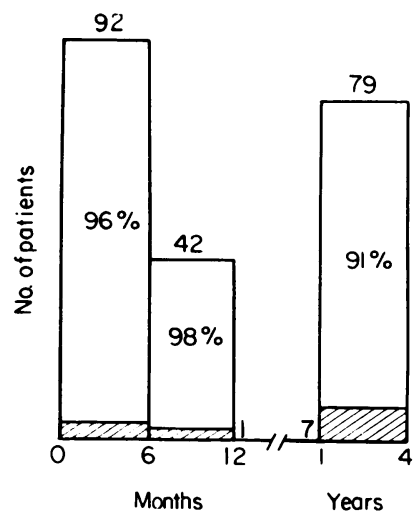

Fig. 2. Number of patients with one or more patent grafts. $\square$, Patients with one or more patent grafts 202, $95 \%$; , patients with occluded grafts 11 . 


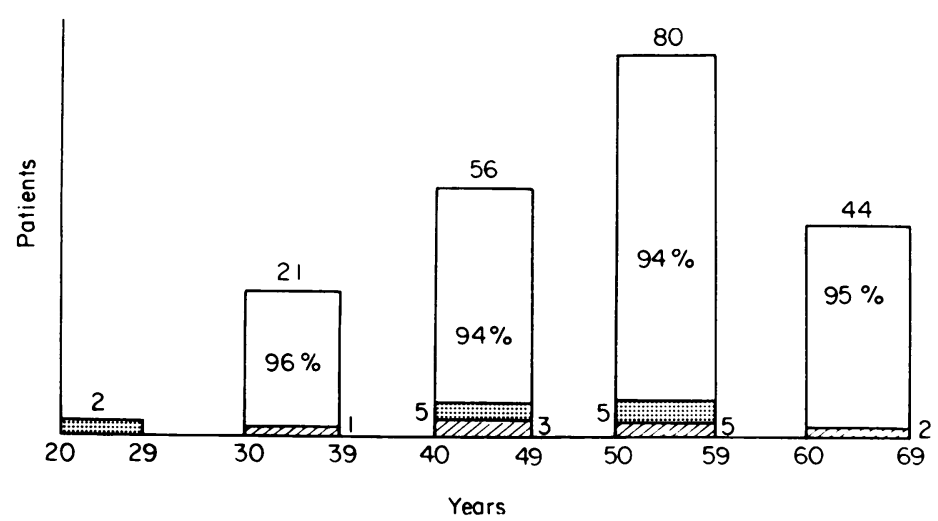

Fig. 3. Effect of age and sex on patency of one or more grafts in patients aged between 27 and 68 years. $\mathscr{W}$, Occluded; $:$, female $10 ; \square$, patent.

\section{Results}

Of the $\mathbf{4 4 1}$ grafts investigated, 388 were shown to be patent, representing an overall patency rate of $88 \%$ (Fig. 1). The patency rate of grafts investigated

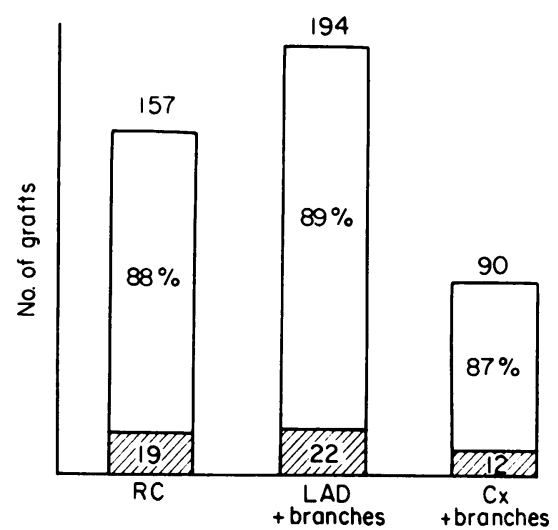

FIG. 4. Patency rate of grafts to different arteries. Ŭ , Occluded 53; $\square$, patent $388,88 \%$. RC, right coronary; LAD, left anterior descending; Cx, circumflex.

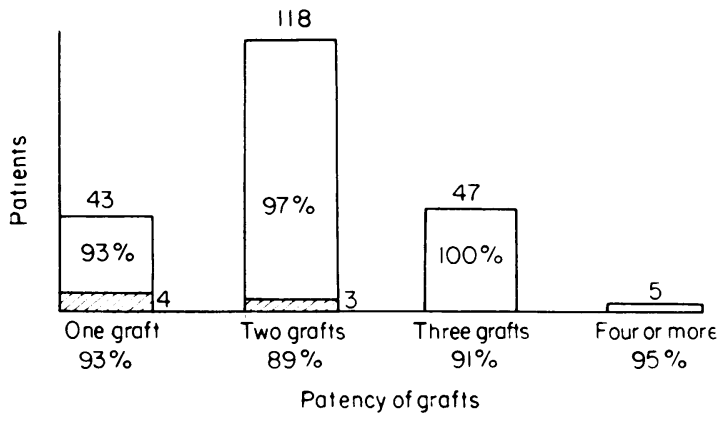

FIG. 5. Patients with one or more patent grafts related to number of grafts. $\square$, One or more grafts patent; $\mathbb{Z}$, all grafts occluded. during the first 6 months was $94 \%$, compared to $83 \%$ for those investigated $1-4$ years after operation (Fig. 1). This difference, however, did not reach statistical significance. Of the patients investigated, $95 \%$ had one or more patent graft (Fig. 2). This was not significantly altered with the age of the patient (Fig. 3) nor the time between operation and investigation (Fig. 2). The patency rate of grafts to different arteries was remarkably similar (Fig. 4). The number of inserted grafts in each patient did not affect the patency rate (Fig. 5). However, as can be expected, $93 \%$ of patients with a single graft had one graft patent compared to $100 \%$ of those with three or more grafts.

Additional endarterectomy (using carbon dioxide in most cases) did not affect the patency rate. Thus, out of eighty-six grafts to endarterectomized arteries seventy-six were patent, representing a patency rate of $88 \%$ which was identical to the patency rate of the 355 grafts to non-endarterectomized arteries (Fig. 6).

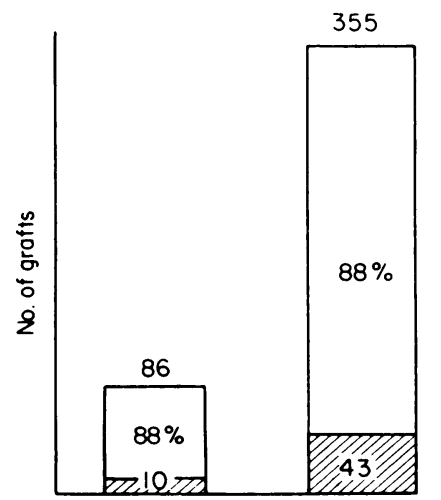

With endorterectomy No endorterectomy

FIG. 6. Effect of endarterectomy on patency rate. $\mathscr{\mathbb { H }}$, Occluded; $\square$, patent. 


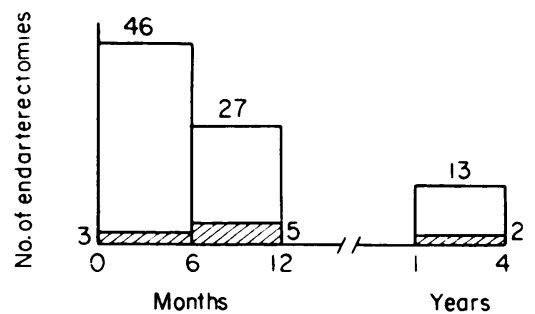

Fig. 7. Patency rate of endarterectomized arteries related to time. $\square$, Patent $76 ; \mathbb{Z}$, occluded 10 .

There was no significant change in patency rate of grafts to endarterectomized arteries with the passage of time (Fig. 7). The run-off of grafts to endarterectomized arteries was judged to be good in $73 \%$ of cases, compared to $76 \%$ for non-endarterectomized arteries (Fig. 8). The patency rate, as well as the runoff of grafts to endarterectomized arteries was similar for the right and left coronary systems (Fig. 9).

The effect of the pre-operative degree of stenosis of the grafted vessel on the patency rate was examined by analysing the patency rate of the grafts to the arteries which were totally occluded before opera-

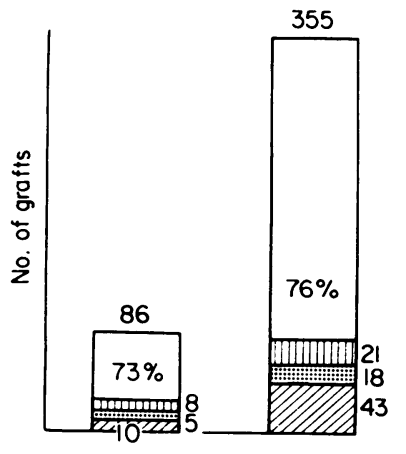

With endarterectomy No endarterectomy

Fig. 8. Effect of endarterectomy on peripheral run-off. $\mathbb{W}$ Occluded; $\mathbb{l}$, moderate; $:$, poor; $\square$, good.

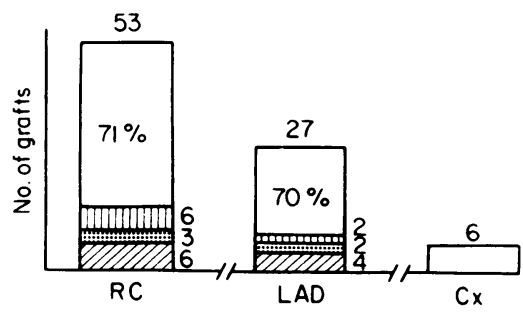

FIG. 9. Run-off in patent grafts to endarterectomized arteries. $\mathbb{Z}$, Occluded; $\mathbb{W}$, moderate; $:$, poor; $\square$, good. $\mathrm{RC}$, right coronary; LAD, left anterior descending; $\mathrm{Cx}$, circumflex.

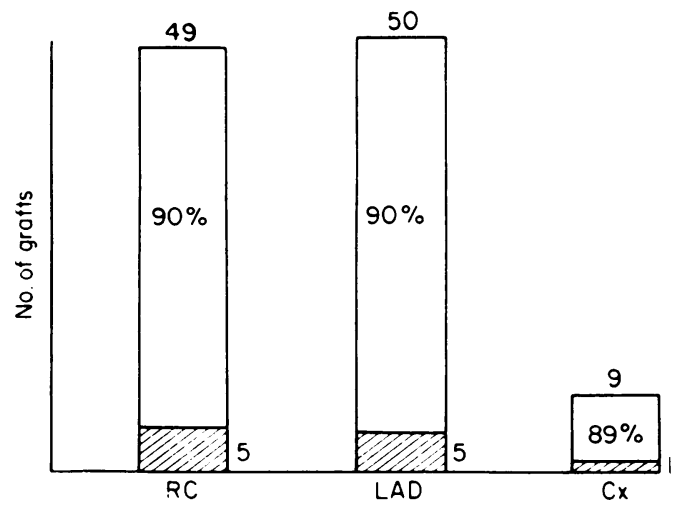

FIG. 10. Patency rate in 108 grafts to vessels totally occluded before operation. $\square$, Patent; $\mathbb{Z}$, occluded. RC, right coronary; LAD, left anterior descending; Cx, circumflex.

tion (Fig. 10). This showed a patency rate of $90 \%$ for grafts to the right coronary and anterior descending, and $89 \%$ for those to the circumflex. This was very similar to the general patency rate.

Analysis of the effect of grafting on the native coronary circulation was made in patients followedup for a minimum period of 6 months (Fig. 11). Progress of the disease proximal to the entry of a patent graft was observed in $16 \%$ of cases, which is similar to progress of the disease in the non-grafted vessels. However, as some of the grafted vessels were totally occluded pre-operatively, and therefore not subject to further progress, it is probable that progress of the disease is faster proximal to the patent graft. Progress of the disease distal to the site of the anastomosis was observed in $3 \%$ of cases. The state

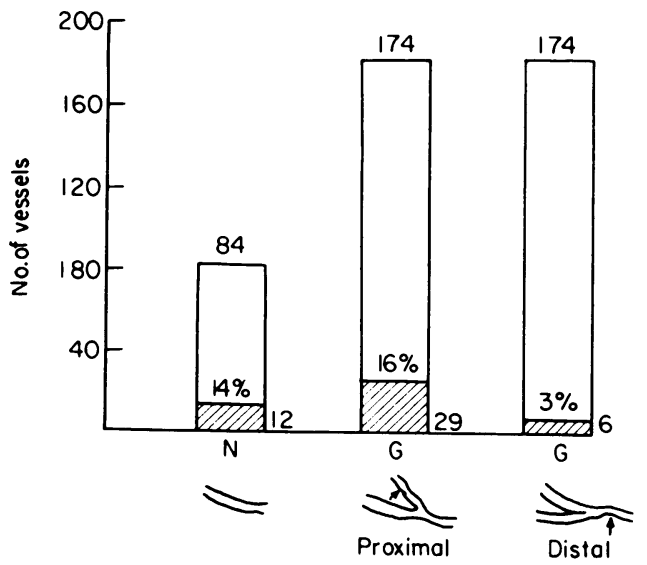

FIG. 11. Effect of grafting on native coronary circulation. Follow-up 6-48 months (mean 20 months), eighty-six patients. N, non-grafted vessel; $\mathrm{G}$, grafted vessel. 


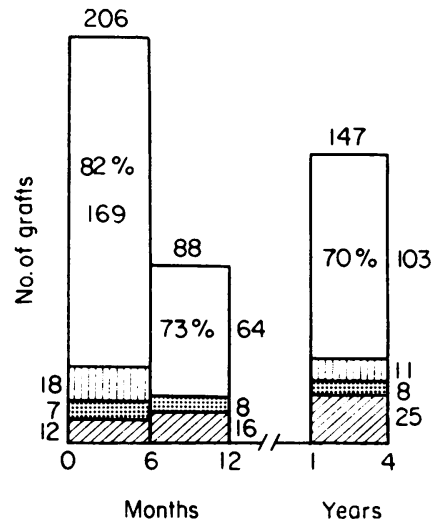

FIG. 12. Assessment of run-off after coronary grafting. $\mathbb{Z}$, Occluded; $\mathbf{l}$, moderate; $:$, poor; $\square$, good.

of the coronary vessels distal to the anastomosis (the run-off) was analysed in relation to time (Fig. 12). This showed $82 \%$ of the run-off grafts investigated early (before 6 months) were judged to be good, compared to $70 \%$ of those investigated late. This difference, however, is not statistically significant.

\section{Discussion}

The present study has shown that saphenous vein $\stackrel{\mathbb{D}}{2}$ bypass grafts continue to function satisfactorily for a $C$. period of up to 4 years. The patency rate does not $\overrightarrow{\vec{F}}$ appear to be influenced by the age or the sex of the patient, the artery grafted, the severity of the lesion pre-operatively, additional endarterectomy nor the interval between operation and re-investigation. Progress of the disease appeared to be slightly accelerated in the segment proximal to a patent graft. Further of studies are required to define the long-term results of the operation.

\section{Acknowledgment}

I would like to acknowledge with thanks the help of Doctors Fawzy, Ahmed, Wesbury and Towers.

\section{References}

Yacoub, M.H., GoldberG, D., Fawzy, E., Raftery, E.B., KNIGHT, E. \& Towers, M. (1974) Late results of direct myocardial revascularisation. Thoraxchirurgie, 22, 590.

Yacoub, M.H., Fawzy, E., Anyanwu, H. \& Towers, M. 음 (1975) Combined gas endarterectomy and coronary artery bypass grafting. A follow-up study. Circulation, 52 (Suppl. 1), 182. 